\title{
The nuclear modification factor of $D$ and $B$ mesons in a field fluctuating quark-gluon plasma at LHC energies
}

\author{
Ashik lkbal Sheikh* \\ Variable Energy Cyclotron Centre, HBNI, 1/AF Bidhan Nagar, Kolkata 700 064, INDIA \\ E-mail: ashikhep@gmail.com

\section{Zubayer Ahammed} \\ Variable Energy Cyclotron Centre, HBNI, I/AF Bidhan Nagar, Kolkata 700 064, INDIA \\ E-mail: za@vecc.gov.in

\section{Munshi Golam Mustafa} \\ Theory Division, Saha Institute of Nuclear Physics, HBNI, 1/AF Bidhannagar, Kolkata 700064 , \\ INDIA \\ E-mail: munshigolam.mustafa@saha.ac.in
}

\begin{abstract}
We have calculated the nuclear modification factor $\left(R_{A A}\right)$ of $\mathrm{D}$ and $\mathrm{B}$ mesons by considering the energy gain due to field fluctuations along with collisional and radiative energy loss of charm and beauty quarks in the hot and dense deconfined medium of quarks and gluons created in relativistic heavy ion collisions. We find a good agreement with the experimentally measured $R_{A A}$ of D and B mesons by ALICE and CMS experiments at $\sqrt{s_{N N}}=2.76 \mathrm{TeV}$ and $\sqrt{s_{N N}}=5.02 \mathrm{TeV}$.
\end{abstract}

The International Conference on B-Physics at Frontier Machines - BEAUTY2018

6-11 May, 2018

La Biodola, Elba Island, Italy

${ }^{*}$ Speaker. 


\section{Introduction}

One of the important features of the Quark-Gluon Plasma (QGP) is the suppression of high energy hadrons compared to the case of $p-p$ collisions, called jet quenching which is because of the energy loss of initial hard partons via collisional and radiative processes inside the deconfined medium. In view of this the energy loss suffered by highly energetic partons, both light and heavy quarks [1-8], in the deconfined QCD medium is a field of immense interest. Heavy quarks are mostly produced in early stage of the heavy ion collisions from the initial fusion of partons. Hence, the total number of heavy quarks becomes frozen at the very early stage in the history of the collisions, which makes them a good probe of the QGP. These heavy quarks immediately after their production will propagate through the dense medium and will start losing energy by elastic collisons and gluon radiations during their path of travel. This energy loss suffered by the heavy quarks is reflected in the transverse momentum spectra and nuclear modification factor (i.e. ratio between the yields in nucleus-nucleus collisions and binary scaled $p-p$ collisions) of heavy mesons. Since QGP is a statistical ensemble of mobile coloured charge particles, it could be characterised by omnipresent stochastic fluctuations. These microscopic fluctuations lead to an energy gain of heavy quarks of all momentum and significantly at the lower momentum [6]. The effect of such fluctuations has an impact on the heavy hadron spectra in the perspective of heavy ion collisions [9].

In this article, we investigate the effect of the chromo-electromagnetic field fluctuations leading to energy gain of heavy quarks in addition to both collisional and the radiative energy loss on the nuclear modification factor for $D$ and $B$ mesons and compared with the measurements of both ALICE and CMS experiments in $P b-P b$ collisions at $\sqrt{s_{N N}}=2.76 \mathrm{TeV}$ and CMS experiment at $\sqrt{s_{N N}}=5.02 \mathrm{TeV}$.

\section{Heavy quark production and fragmentation}

The heavy quarks in $p-p$ collisions are mainly produced by fusion of gluons or light quarks. The production cross section for heavy quarks in $p-p$ collisions is calculated at orders higher than Leading Order (LO) using CT10 parton distribution function [10]. For heavy ion collisions, the shadowing effect is taken into account by using the NLO parameters of spatially dependent EPS09 [11] nuclear parton distribution function and obtained the spectrum in $\mathrm{Pb}-\mathrm{Pb}$ collisions by including the the momentum loss $\Delta p_{T}$ in the calculated differential cross section [8]. For fragmentation of $c$ quarks into $D$-mesons and $b$ quarks into $B$-mesons, the Peterson fragmentation function [12] with parameters $\varepsilon_{c}=0.016$ for $c$ quarks and $\varepsilon_{b}=0.0012$ for $b$ quarks is used.

\section{QGP Evolution and initial condition}

We consider a heavy quark, which is being produced at a point $(r, \phi)$ in heavy ion collisions and propagates at an angle $\phi$ with respect to $\hat{r}$ in the transverse plane. So, the path length $L$ covered by the heavy quark inside the medium is given by, $L(r, \phi)=\sqrt{R^{2}-r^{2} \sin ^{2} \phi}-r \cos \phi$, where $R$ is the radius of the colliding nuclei [13]. 
We estimate $\langle L\rangle=6.14 \mathrm{fm}$ for central $\mathrm{Pb}-\mathrm{Pb}$ collisions. The effective path length of heavy quark of transverse mass $m_{T}$ and transverse momentum $p_{T}$ in the QGP of life time $\tau_{f}$ is obtained as $L_{\text {eff }}=\min \left[L, \frac{p_{T}}{m_{T}} \times \tau_{f}\right]$. We consider the medium evolution as per the isentropic cylindrical expansion using Lattice QCD equation of state along with hadronic resonance as a simplistic approach as discussed in Ref. [14].

\section{Energy Loss: Collisional Loss (Peigne and Peshier (PP) Formalism) and Radiative Loss (Abir, Jamil, Mustafa and Srivastava (AJMS) Formalism)}

The most detailed calculation of collisional energy loss $d E / d x$ was made by Brateen and Thoma [1], which was based on their previous QED calculation of $d E / d x$ for muon [15]. This calculation of Brateen and Thoma for $d E / d x$ is based on an assumption that $q \ll E$, where $q$ is the momentum exchange in elastic collisions and $E$ is the energy of the heavy quark. This assumption is not appropriate in the domain $E \gg M^{2} / T$, where $M$ is the mass of the heavy quark and $T$ is the temperature of the medium. The improved differential energy loss expression, valid for $E \gg M^{2} / T$, is given by Peigne and Pashier [5].

The most important and dominant way of energy loss from a fast parton inside the QGP is due to gluon radiation [7, 8]. Many authors [2-4,7] estimated the energy loss with various ingredients and kinematical conditions. In Refs. [2,3] the soft gluon emission by heavy quarks was estimated which was found to suppress compared to the light quarks due to the mass effect, known as dead cone effect. The radiative energy loss induced by the medium due to the dead cone effect was limited only to the forward direction. In Ref. [4] by relaxing some of the constraints imposed in Refs. [2,3], e.g., the gluon emission angle and the scaled mass of the heavy quark with its energy, a generalised dead cone was obtained which led to a very compact expression for the gluon emission probability off a heavy quark. Based on the generalised dead cone approach and the gluon emission probability [4], AJMS [7] computed the heavy quark radiative energy loss. Later a kinematical correction was made in Ref. [8].

\section{Energy gain by chromo-electromagnetic fields fluctuations: Chakraborty, Mustafa and Thoma (CMT) Formalism}

The energy loss calculations both collisional and radiative of heavy quarks in the QGP were obtained by treating the QGP medium without any microscopic fluctuations. However, QGP being the statistical system, it is characterised by stochastic chromo-electromagnetic field fluctuations. A quantitative estimate of the effect of the microscopic electromagnetic fluctuations on the propagation of a heavy quark was done using semiclassical approximation by CMT [6]. This was found to lead to an energy gain of the heavy quark caused by the statistical change in the energy of the moving parton in the QGP due to such fluctuations in fields as well as the velocity of the particle under the influence of this field. The leading-log (LL) contribution of the energy gain was obtained [6] as

$$
\left(\frac{d E}{d x}\right)_{\mathrm{fl}}^{\mathrm{LL}}=2 \pi C_{F} \alpha_{s}^{2}\left(1+\frac{n_{f}}{6}\right) \frac{T^{3}}{E v^{2}} \ln \frac{1+v}{1-v} \ln \frac{k_{\max }}{k_{\min }},
$$


where $k_{\min }=\mu_{g}=$ Debye mass and $k_{\max }=\min \left[E, \frac{2 q(E+p)}{\sqrt{M^{2}+2 q(E+p)}}\right]$ with $q \sim T$ is the typical momentum of the thermal partons. One can physically interpret this energy gain of a heavy quark as it absorbs gluons during its propagation.

\section{Results and Discussions}

In Fig. 1 and Fig. 2 we have displayed the nuclear modification factor $R_{A A}$ for $D^{0}$-meson in $(0-$ $10) \%$ and $(0-100) \%$ centrality, respectively, in $P b-P b$ collisions, considering both collisional and radiative energy loss along with the energy gain due to the field fluctuations and compared with ALICE [16] and CMS [17] data. We observe that the measured data within their uncertainties can be nicely described with the energy gains due to the field fluctuations in addition to both collisional and radiative losses. The field fluctuations are found to play an important role in the phenomenology of the heavy quark jet quenching observed in the LHC energies.

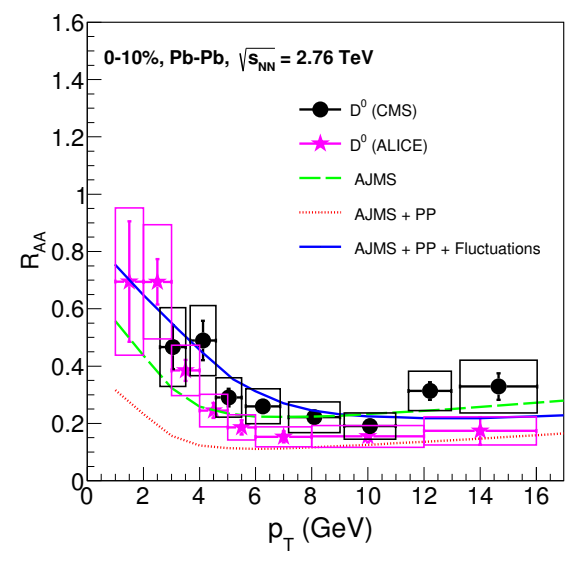

Figure 1: Nuclear modification factor $R_{A A}$ of $D^{0}$-meson as a function of transverse momentum $p_{T}$ for $(0-10) \%$ centrality at $P b-P b$ collisions at $\sqrt{s_{N N}}=2.76 \mathrm{TeV}$.

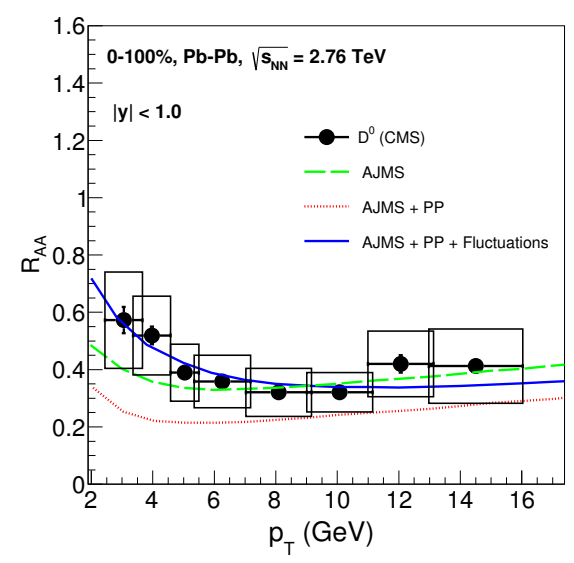

Figure 2: Nuclear modification factor $R_{A A}$ of $D^{0}$-meson as a function of transverse momentum $p_{T}$ for $(0-100) \%$ centrality in $P b-P b$ collisions at $\sqrt{s_{N N}}=2.76 \mathrm{TeV}$.

Fig.3 displays the nuclear modification factor $R_{A A}$ of $D^{0}$-meson as a function of transverse momentum $p_{T}$, obtained using collisional (PP), radiative (AJMS) energy loss and the energy gain due to field fluctuations in $P b-P b$ collisions at $\sqrt{s_{N N}}=5.02 \mathrm{TeV}$. The experimental data is obtained from the CMS collaboration [17]. The $R_{A A}$ is in better agreement with data within their uncertainties only at low $p_{T}$ when the fluctuations are included.

In Fig.4 the nuclear modification factor $R_{A A}$ for $B^{+}$-meson in $P b-P b$ collisions at $\sqrt{s_{N N}}=$ $5.02 \mathrm{TeV}$ is displayed by considering both collisional and radiative energy loss along with the effect of the field fluctuations and compared with CMS data [17]. The radiative energy loss itself produces a small suppression. The field fluctuations along with the radiative and collisional losses produce larger suppression compared to radiative alone. The experimental uncertainties are still large to draw conclusion on the effect of field fluctuations in the beauty energy loss.

In conclusion, the calculated nuclear modification factors $R_{A A}$ for $D$ mesons are found to agree quite well with the measured $R_{A A}$ of $D$ mesons by CMS and ALICE experiments at LHC, if the 


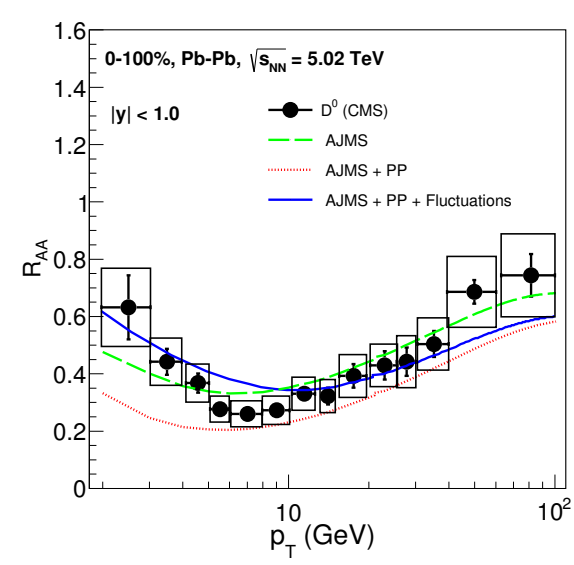

Figure 3: Same as Fig.2 but at $\sqrt{s_{N N}}=$ $5.02 \mathrm{TeV}$.

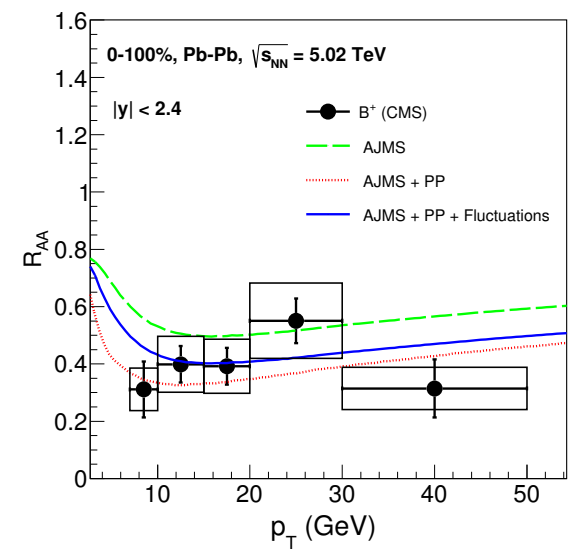

Figure 4: Same as Fig.2 but for $B^{+}$-meson at $\sqrt{s_{N N}}=5.02 \mathrm{TeV}$.

energy gain due to the field fluctuations is taken into account in addition to the collisional and radiative loss in the medium. The experimental uncertainties are large for $B$ mesons to conclude anything on the effect of field fluctuations. More precise measurements might shed light on it.

\section{References}

[1] E. Brateen and M.H. Thoma, Phys. Rev. D 44, R2625 (1991); M.H. Thoma and M. Gyulassy, Nucl. Phys. B351 (1991) 491-506.

[2] Y. L. Dokshitzer and D. E. Kharzeev, Phys. Lett. B519, 199 (2001).

[3] Y. L. Dokshitzer, V. A. Khoze, and S. I. Troian, J. Phys. G17, 1602 (1991).

[4] R. Abir, C. Greiner, M. Martinez, M. G. Mustafa, and J. Uphoff, Phys. Rev. D 85, 054012 (2012).

[5] S. Peigne and A. Peshier, Phys. Rev. D 77, 114017 (2008).

[6] P. Chakraborty, M.G. Mustafa and M.H. Thoma, Phys. Rev. C 75, 064908 (2007).

[7] R. Abir, U. Jamil, M.G Mustafa and D.K Srivastava, Phys. Lett. B 715 (2012) 183.

[8] K. Saraswat, P. Shukla and V. Singh, Nucl. Phys. A 934 (2015) 83; Nucl. Phys. A 961 (2017) 169.

[9] A.I. Sheikh, Z. Ahammed, P. Shukla and M.G. Mustafa, arXiv:1711.06245 (2017).

[10] H.L. Lai et. al., Phys. Rev. D 82 (2010) 074024.

[11] K.J. Eskola, H. Paukkunen and C.A. Salgado, J. High Energy Phys. 0904 (2009) 065.

[12] C. Peterson, D. Schlatter, I. Schmitt and P. Zerwas, Phys. Rev. D 27 (1983) 105.

[13] B. Muller, Phys. Rev. C 67 (2003) 061901.

[14] X. Zhao and R. Rapp, Nucl. Phys. A 859 (2011) 114.

[15] E. Brateen and M.H. Thoma, Phys. Rev. D 44, 1298 (1991).

[16] ALICE Collaboration, JHEP 1603 (2016) 081, 2016.

[17] CMS Collaboration, CMS-PAS-HIN-15-005; Phys.Lett. B 782 (2018) 474; Phys. Rev. Lett. 119, 152301 (2017). 